\title{
On the Recognition and Enforcement of Foreign Civil and Commercial Judgments—-the Coordination Between the Parties' Autonomy and the Public Order
}

\author{
Yunxia Sun 1, 2, a, Haifeng Liu 1, b, Jinlei Li ${ }^{1, c}$ and Peng Zhang 2, d \\ ${ }^{1}$ Tianjin University Renai College, Tianjin 301636 China; \\ ${ }^{2}$ Tianjin Wisely Law Office, Tianjin 300202 China. \\ aavasunsyx@126.com, bbilly2006@126.com, clijinlei_86@163.com, dlaw_person@126.com
}

Keywords: Foreign civil and commercial judgments; recognition and enforcement; indirect jurisdiction; parties' autonomy; application of law; public order.

\begin{abstract}
The factors that affect the recognition and enforcement of foreign civil and commercial judgments include the jurisdiction of the court and the public order of the requested country. Especially when the foreign judgment is made on the basis of the parties' choice of court and the choice of law, it is necessary to consider the coordination between the party autonomy and the public order. In the design of the legal system of the recognition and enforcement foreign judgment, China should take the parties' autonomy of choices of law that is given by the Chinese procedural law and conflict laws into consideration, to restrict the reservation of public order correspondingly, in order to achieve the consistency of the recognition and enforcement of foreign judgments in the system.
\end{abstract}

\section{外国民商事判决的承认与执行问题探析一一当事人意思自治与公共秩序的协调}

\author{
孙云霞 ${ }^{1,2}$, 刘海峰 ${ }^{1}$, 李金䂞 ${ }^{1}$, 张鹏 $^{2}$ \\ 天津大学仁爱学院, 天津 中国; \\ 2. 天津华盛理律师事务所, 天津 中国
}

摘 要: 影响一国法院承认与执行外国民商事判决的因素包括判决做出法院的管辖权以及被请 求承认与执行地公共秩序等。当外国判决的做出是基于当事人选择法院以及选择法律等前提 时, 要考虑执行地法律对当事人的意思自治与公共秩序的协调。中国应在审查外国判决的法 律制度设计中考虑到中国的诉讼法及冲突法中赋予当事人的意思自治选择权, 对公共秩序保 留的适用进行相应限制, 以便做到外国判决承认与执行制度中的一致性。

关键词: 外国民商事判决; 承认与执行; 意思自治; 间接管辖权; 法律适用; 公共秩序.

\section{1. 前言}

外国民商事判决的承认与执行, 既是国际民商事争议解决的最终环节, 也是国际民商事秩序 得以维护和健全的必要保障。[1] 国际民商事案件的当事人在外国取得确权判决或者有执行性 的判决后, 需要在承认地以判决确认身份或权利行事, 或需要在执行地实现该权利, 从而向 特定的一国法院申请承认和执行, 是发生外国民商事判决承认与执行的最主要的原因。国际 民商事判决的承认与执行, 既涉及到受理案件法院的管辖权, 又与该法院审理案件的冲突法、 程序法以及法院最终适用的准据法密切相关, 还受限于申请承认与执行地的公共秩序。课题 研究外国判决承认与执行背景下的当事人意思自治与法院地公共秩序保留之间的协调, 可以 为我国在国际私法制度设计中提供有益借鉴, 有助于实现我国判决承认与执行中法律制度的 一致性。 


\section{2. 对外国判决承认与执行审查的内容与标准}

主权国家基于属地管辖原则对其本国领域内的所有人、事、物、行为享有独占的管辖权，一 国法院已生效民商事判决在本国境内冊庸置疑拥有司法确定性和强制执行力, 而该判决在外 国是否具有司法确定性和强制执行力则要看相关国家是否予以承认和执行。现代社会中，任 何一个国家都无法脱离国际社会独立存在，一国法院已生效判决在另一国能否得到承认问题 将可能影响到当事人之后在该另一国的能力与权利。如一国法院所做出的离婚判决，一般将 被其他国家所承认，该当事人在承认国可以单身身份再次缔结婚姻。

被请求国法院审查外国民商事判决的内容和标准根据各国国内法及其参加的国际条约的不同 而有所区别，但审查的内容都包含做出判决的外国法院对该案是否有管辖权、该判决的作出 是否给予被告以适当的抗辩机会、判决作出程序是否正义、判决本身是否已生效、该判决是 否违背被请求法院地国的公共秩序等方面。对于双方当事人协议选择法院作出的判决, 选择 法院协议本身的效力是审查作出判决的外国法院对案件管辖权的重要内容。

2.1. 海牙《协议选择法院公约》的相关规定

2005 年海牙《协议选择法院公约》对协议选择法院条款的效力与承认和执行外国法院判决之 间的某种联系进行了规定，公约第一条、第二条规定协议选择法院的范围及适用除外，第八 条规定经当事人协议选择的法院作出的国际民商事判决应予承认和执行所必备的条件，第九 条规定一国法院拒绝承认和执行经由当事人协议选择的法院作出的国际民商事判决的事由。

2.1.1协议选择法院的范围

根据海牙《协议选择法院公约》，对于国际性民商事案件，已经由当事人订立排他性选择法 院协议确立管辖权的法院作出的判决, 可寻求被承认与执行。但如果该国际民商事关系属于 消费合同、雇佣合同，或涉及自然人的身份与法律能力、扶养义务婚姻家庭、遗嘱和继承、 破产、旅客和货物的运费、海事污染及海事请求权的责任限制，共同海损，以及紧急拖航和 海上救助、竞争法事项、核能损害责任、自然人引起的人身损害赔偿、侵权诉讼、不动产物 权、决定法人有效的决定、版权和邻接权之外的知识产权的有效性等内容则不属于公约认可 的协议选择法院的范围。

2.1.2拒绝承认与执行判决的情形

被请求国必须承认与执行由排他性选择法院协议指定的外国法院所作出的已生效判决，并不 得就原审法院作出判决的合理性进行审查, 被请求国法院应被经选择的原审法院认定的事实 的约束，除非该判决系缺席作出。

只有当该外国判决有下列情形时，被请求国方可拒绝承认或执行：根据被选择法院国家的法 律该法院选择协议无效、根据被请求国的法律一方当事人无缔结协议的能力、诉讼中未给被 告充分的时间并以一定方式通知被告以便安排答辩、违法被请求国通司法文书送达的基本原 则、诉讼程序存在欺诈、与被请求国就相同当事人间的争议所做出的判决不一致、与另一国 的在先判决不一致、承认或执行判决将明显违背被请求国的公共政策（包括被请求国的程序 公正原则）。

\section{2. 中国双边司法协助条约及国内诉讼法的相关规定}

中国迄今为止已经与 36 个国家签订了民事、民事和商事、民事和刑事或者民事、商事和刑事 司法协助条约或协定，除与比利时和阿根廷签订的协定（条约）尚未生效外, 其他均已生效。 在已经生效的 34 个条约（协定）中，除与泰国、新加坡和韩国签订的条约外，其他都包含有 相互承认和执行对方法院裁判的规定。中国《民事诉讼法》及其司法解释关于外国民事判决 的承认与执行也做了原则性规定。综合各双边司法协助条约及中国涉外诉讼法的规定，对于 外国民商事判决的承认和执行包括应予以承认和执行的外国民商事判决的范围、拒绝承认与 执行的情形以及以下内容。

\subsection{1承认和执行判决的范围}

中国《民事诉讼法》规定，中国人民法院作出的发生法律效力的判决、裁定，如果被执行人 或者其财产不在中华人民共和国领域内, 当事人请求执行的, 可以由当事人直接向有管辖权 
的外国法院申请承认和执行，也可以由人民法院依照中华人民共和国缔结或者参加的国际条 约的规定, 或者按照互惠原则, 请求外国法院承认和执行。外国法院作出的发生法律效力的 判决、裁定，需要中华人民共和国人民法院承认和执行的，可以由当事人直接向中华人民共 和国有管辖权的中级人民法院申请承认和执行，也可以由外国法院依照该国与中华人民共和 国缔结或者参加的国际条约的规定，或者按照互惠原则，请求人民法院承认和执行。 双边司法协助条约中大都规定，一方应当根据本条约规定的条件，采取措施承认和执行另一 方法院在民事和商事案件中作出的裁判文书及审理刑事案件的法院就民事权利作出的裁判文 书，但关于遗嘱或者继承、破产、社会保障及除涉及生活费的保全措施或者临时措施除外。 2.2.2拒绝承认与执行判决的情形

被请求方在下列情况下可以拒绝承认和执行外国判决：根据裁判文书作出方的法律，该裁判 文书尚未生效或者不具有执行效力; 根据被请求方的法律, 作出裁判文书的法院无管辖权; 根据裁判文书作出方的法律，缺席的败诉方当事人未经合法传唤，或者无诉讼行为能力的当 事人没有得到适当代理；被请求方法院正在审理相同当事人之间就同一事实和标的提起的案 件, 或者已经作出生效裁判文书, 或者已经承认或者执行第三国法院作出的裁判文书; 判决 所支持的诉讼请求违反被请求方现行法律，或与被请求方的宪法原则、主权、安全或公共秩 序相悖。

\section{3. 海牙《协议选择法院公约》与中国司法协助条约之比较}

海牙《协议选择法院公约》与中国司法协助条约关于可以申请承认和执行的范围区别较大, 其主要原因在于海牙《协议选择法院公约》关于请求承认与执行的外国法院判决主要限于可 以协议选择法院的民商事领域，关于消费合同、雇佣合同、涉及人的身份与能力、婚姻家庭、 继承、侵权等领域，公约并不支持当事人之间就管辖权进行选择，中国司法协助条约中承认 与执行外国判决的范围除了当事人意思自治选择的法院作出的判决, 一国法院根据其他管辖 原则作出的判决同样属于可以请求承认与执行的范围。

关于拒绝承认与执行的情形, 二者均包括判决作出法院无管辖权、判决作出程序缺陷、申请 承认与执行程序不符合被请求国法律规定、违背被请求国公共秩序、诉讼竞合等情形。但是 对作出判决的外国法院是否有管辖权的审查标准, 海牙《协议选择法院公约》和中国相关司 法协助条约规定的审查依据是截然不同的。海牙《协议选择法院公约》第九条第一款规定， 根据被选择法院国家的法律, 选择法院的协议无效将导致原审法院对该案不享有管辖权; 中 国的司法协助条约则规定根据被请求方的法律来审查作出裁判文书的法院是否有管辖权。

\section{3. 公共秩序在外国法院判决承认与执行中的作用}

\section{1 公共秩序保留在国际民商事判决中的应用}

公共秩序在不同国家的立法中有多种表述，包括 “公共利益”、“基本政策”、“法律的基 本原则或国家主权、安全、社会公共利益”、“社会公共利益”等。自意大利巴托鲁斯的 “法 则区别说” 产生至今约七百年，公共秩序并没有随着岁月的沉淀而形成国际私法基本制度所 应具有的公认的制度内涵, 各国在公共秩序的规定上如此多样以致其本来面目至今仍扑朔迷 离。[2]英国著名冲突法学者莫里斯认为, 冲突法中保留公共秩序是必要的, 但给这种保留做 一个明确的界限既没有必要也无法实现。随着各国在冲突法领域的改革，对当事人规避法律 以及对法律进行选择的方式和内容进行更加细化地规定, 立法手段更先进有效, 更多地通过 选择性及重叠适用的冲突规范的使用, 以及相关配套制度的完善, 使得公共秩序保留应用的 余地越来越小。

公共秩序保留作为国际私法基本制度之一，因其内涵的丰富和外延的模糊在法律选择和外国 法适用中有比其他制度具有更强的灵活性和随意性。在法律选择环节，当内国法院根据本国 冲突规范的指引本应适用外国法时, 如果该外国法的内容与内国公共秩序相冲突, 则可以公 共秩序保留为由不予适用该外国法, 此时公共秩序保留被审理案件的法院用以保护法院地的 公共秩序。各国及一些国际立法都将公共秩序作为法律选择的“安全阀”来使用。 
而公共秩序在外国判决的承认与执行领域同样可以作为衡量外国判决应否予以执行的参照标 准。1968 年布鲁塞尔《民商事司法管辖权和判决执行公约》、1972 年海牙《关于承认与执行 外国民事和商事判决的公约》和 1988 年卢加诺《民商事司法管辖权和判决执行公约》都规定 一国可以公共秩序为由拒绝承认和执行外国判决。

承认与执行外国判决的问题与冲突法机制有所不同, 它要调整的已不是当事人之间的权利纷 争, 而主要是外国法院判决本身的效果, 因此要解决的一个主要问题就是承认或执行外国判决 在后果上是否会违反本国的公共秩序。[3]

3.2 公共秩序在外国法院判决承认与执行中的作用及趋势

公共秩序在解决国际民商事冲突中的 “安全阀” 功能的实现在法律适用中仅是一个层面的表 现, 因为国际民商事法律冲突的最终解决往往并非随着法律选择的完成而结束的。对于那些 需要在外国得到承认和执行的判决而言, 只有通过相关国家间的相互承认与执行才能最终解 决国际民商事纠纷, 化解法律冲突。而根据 “场所支配行为” 这一古老的原则, 经由承认和 执行地法院以及公共秩序进行衡量, 进行承认并协助执行对于外国判决的实现来说是更为重 要的。

对于已经生效的外国法院的判决, 有学者认为对其承认和执行只是承认已获得外国法承认的 权利, 这种观点可认为源自于既得权说。在法院审理案件过程中已经以法院地的公共秩序进 行了审查, 外国法已经发挥了起作用, 在外国作出判决后就成了既成事实。承认与执行阶段 法院地与案件实体内容的联系本就比审判地法院少, 如再以公共秩序为由拒绝承认和执行在 程序上并无瑕疪的外国判决, 将会使已经确定判决和已经确定的民商事关系重新回到不确定 状态，增加司法成本，因此此时不适用公共秩序保留制度。

\section{4 当事人意思自治在外国判决中的作用}

在国际民商事领域通过诉讼解决纠纷的过程中, 当事人意思自治原则主要体现在两个方面, 一是当事人双方通过法院选择协议选择审理案件的法院, 二是双方通过法律选择条款选择解 决纠纷所应适用的实体法。在国际民商事领域, 法院审理国际民事案件适用法院地的诉讼程 序法，选择受理案件的法院实质上同时实现了对诉讼程序法选择。

\section{1 关于国际民商事管辖权确定中的意思自治}

而当事人对法院的选择与法律适用的选择是相互影响、互为制约的。不同国家以及同一国家 的不同时期对当事人法院选择以及法律选择的范围和限制有所不同。

根据 2005 年海牙《协议选择法院公约》第二条规定, 当事人可以通过协议选择法院的方式确 定管辖权的国际民商事纠纷不包括消费合同、雇佣合同，或涉及自然人的身份与法律能力、 扶养义务婚姻家庭、遗嘱和继承、破产、旅客和货物的运费、海事污染及海事请求权的责任 限制, 共同海损, 以及紧急拖航和海上救助、竞争法事项、核能损害责任、自然人引起的人 身损害赔偿、侵权诉讼、不动产物权、决定法人有效的决定、版权和邻接权之外的知识产权 的有效性等。

中国《最高人民法院关于适用〈中华人民共和国民事诉讼法〉的解释》规定涉外合同或者其他 财产权益纠纷的当事人, 可以书面协议选择被告住所地、合同履行地、合同签订地、原告住 所地、标的物所在地、侵权行为地等与争议有实际联系地点的外国法院管辖, 属于中华人民 共和国法院专属管辖的案件, 当事人不得协议选择外国法院管辖。

涉外民事案件中如当事人之间不存在选择中华人民共和国法院管辖的协议、被告提出案件应 由更方便外国法院管辖的请求、不属于中华人民共和国法院专属、不涉及中华人民共和国国 家及具有中国国籍的主体的、主要事实不是发生在中华人民共和国境内, 且案件不适用中华 人民共和国法律等情形的, 人民法院可以裁定驳回原告的起诉, 告知其向更方便的外国法院 提起诉讼。

中国的双边司法协助条约中关于管辖权的内容往往规定, 一国法院有权对在提起诉讼时被告 在该方境内有住所或者居所或分支机构、被告已经接受该方法院的管辖、合同在该方境内签 
订或应当或已经在该方境内履行或者标的物在该方境内、侵权行为或者其结果发生在该方境 内、扶养债权人在提起诉讼时在该方境内有住所或者居所、不动产位于该方境内、在该方境 内有住所当事人提起身份关系的诉讼等行事管辖权。从被告接受该方法院管辖这一款来看, 可以视为双方当事人默示协议选择了该方法院管辖, 我们可以将其归入广义协议确定法院管 辖权的范畴, 如果一方当事人向中国法院起诉, 另一方当事人接受法院管辖并不提出管辖权 异议，此时根据中国关于管辖权的规定，只要中国是被告住所地、合同履行地、合同签订地、 原告住所地、标的物所在地、侵权行为地等与争议有实际联系地点之一, 甚至不局限于以上 实际联系, 只要受理案件法院愿意, 都可以行事管辖权。

尽管中国法律规定的当事人选择法院的范围受实际联系的局限，但这限制仅仅限制了当事人 协议选择法院的范围, 并未对可以选择法院的具体民商事关系的范围进行细分。根据中国诉 讼法及中国签订的双边司法协助条约, 中国关于协议选择规定的范围与《协议选择法院公约》 相比更为宽泛和笼统, 合同和其他财产权益纠纷作为一个概括性规定具有模糊的边界, 并未 像《协议选择法院公约》一样通过列举的方式将与人的身份和能力、侵权、消费者权益保护 等有关的财产权益保护等内容规定为例外条款, 而且 “被告接受” 这一可视为协议管辖的方 式从一定意义上来看, 更是在可供选择的法院和可以选择法院的民商事关系的范围这两个问 题上无限扩大了协议管辖的范围。

\section{2 法律选择中的意思自治}

意思自治原则作为国际私法领域法律选择原则之一, 在各国立法和司法实践中拥有的影响力 逐渐超过最密切联系原则, 成为合同领域乃至侵权领域的优先原则。法律选择条款中意思自 治原则的发展趋势, 通过中国《涉外民事关系法律适用法》与《民法通则》第八章中，允许 当事人对法律适用进行选择的条款数量的对比, 可以明显看出意思自治原则的范围越来越广 泛。《涉外民事关系法律适用法》允许当事人进行协议选择法律的条款包括代理、信托、仲 裁协议、夫妻财产关系、协议离婚、动产物权、运输中动产物权、合同、消费者合同、侵权 责任、产品责任、不当得利和无因管理、知识产权及其侵权等等共十四项内容, 而《民法通 则》第八章仅规定涉外合同的当事人可以选择处理合同争议所适用的法律这一条允许当事人 协议选择的法律选择条款。

允许当事人在纠纷发生前或纠纷发生后对法律适用进行选择有利于及时有效地解决国际民商 事法律纠纷, 有条件地扩大当事人可以进行选择的民事领域的范围对加速解决国际民事纠纷 无疑是有积极意义的。尽管法律赋予了当事人更大的选择法律的自由, 但当事人要真正实现 这个权利有时也要受制于法院地冲突规范的具体规定。

4.3 选择法院对法律选择的影响

管辖权确定中的当事人意思自治原则与法律选择领域当事人意思自治原则的内容、范围和限 制虽然有所区别, 但二者的应用最终都将影响到案件实体问题的法律适用, 而且当事人对法 院的选择将会影响到法律选择的效力。管辖权选择中的意思自治影响法律选择中意思自治的 情形主要有三种情形。当事人协议选择的法院根据法院地的法律概念对其受理的涉外民商事 案件进行识别后, 根据法院地冲突规范的指引寻找准据法, 其一, 如该冲突规范的规定允许 当事人协议选择法律, 且当事人协议选择的法律与法院地公共秩序不冲突, 则法院可以适用 当事人协议选择的法律; 其二, 该冲突规范的规定允许当事人协议选择法律, 但当事人协议 选择的法律与法院地公共秩序冲突, 则法院可以公共秩序保留为由不适用用当事人协议选择 的法律; 其三, 如果法院地冲突规范不允许当事人对所应适用的法律进行选择, 那双方当事 人的选择就是无效的, 法院将根据冲突规范的指引另行寻找准据法。

\section{5 当事人意思自治与被申请执行地法院地公共秩序的协调统一}

在世界经济全球化化及中国与亚非拉国家共建 “一带一路” 的大背景下，全球及区域间民商 事关系国际化态势发展迅猛, 必须充分发挥国际私法各种制度在解决国际民商事法律冲突和 
维护国际民商事新秩序中的积极作用。对于已经外国法院作出的民商事判决, 中国应更谨慎 地对待, 处理好公共秩序与外国判决的承认与执行的关系。

5.1 被请求国审查当事人选择法院协议效力的依据

不同于海牙《协议选择法院公约》根据被选择法院国家的法律审查当事人选择法院协议效力 的做法, 中国法院审查外国判决时依据被请求国即中国法律来审查当事人协议选择法院的行 为是否有效。因此如果双方当事人协议选择的外国法院根据法院地关于管辖权的规定取得管 辖权并受理案件作出了生效判决, 该判决如需到中国申请承认与执行时, 中国法院在审查作 出该判决的法院是否有管辖权时需要依中国关于管辖权的规定来判断, 即中国判断间接管辖 权的依据是中国诉讼法。实践中各国在承认与执行外国法院民商事判决的过程中，审查间接 管辖权的依据主要有两种, 一是以作出判决法院所在国的法律为标准, 二是以被申请国法律 为标准。依原审国法律为标准在现实操作中存在许多缺陷, 如被申请国法院要掌握所有相关 外国管辖权的法律规定、依相同标准审查相同案件的管辖权造成司法重复等，该方法操作性 不强, 不为大多数国家采纳。以被申请国法律为标准审查原判决国法院管辖权的适当与否, 这是大多数国家在没有双边协议或条约时的通常做法。[4]因此中国在双边司法协助条约中大 多采取这种做法并无不当。实践中各国也经常以原审国法院没有接管辖权为由拒绝承认与执 行外国判决的情形。

\section{2 公共秩序保留制度与当事人意思自治的协调}

尽管原审国直接管辖权与被请求国间接管辖权的冲突不可避免, 原审国公共秩序与被请求国 公共秩序之冲突也时会发生, 但一国内公共秩序的内涵与当事人意思自治原则在管辖权和法 律适用领域实现协调统一却是可以实现的。

如前所述，在国际民商事领域管辖权的确定这个问题上，中国的意思自治原则主要体现为有 条件的意思自治, 即以实际联系为意思自治的前提，但原告向一国法院起诉而被告接受的情 况实际上拓展了实际联系原则, 使得无实际联系的法院可能也有可能因被告接受而取得案件 管辖权, 所以尽管协议管辖权的确定表面上看起来需要与案件有实际联系，但实质上并不严 格受实质联系的限制; 而在法律选择领域的意思自治中国则通过《涉外民事关系法律适用法 司法解释（一）》明确了一方当事人以双方协议选择的法律与系争的涉外民事关系没有实际 联系为由主张选择无效的人民法院不予支持，实际上是放弃了 “实质性联系” 标准，这符合 国际社会的立法趋势。[5]因此申请中国承认与执行的外国法院判决极有可能是原审法院与案 件也无实际联系, 判决案件所依据的准据法也与案件无实际联系。发生这种情形, 在不考虑 公共秩序的情况下，根据中国承认与执行外国判决的范围和拒绝承认与执行的相关规定，该 判决并不会因为原审法院没有管辖权构成拒绝承认与执行的条件。而既然向中国法院提出承 认和执行的申请, 则该案件与中国一定有一定的联系, 此时发生判决的程序或内容与被申请 国即中国公共秩序冲突的可能性将大大增加。

各国一般允许选择法院和法律的领域都与合同有关，而合同作为一种民事关系的形式实质上 是对法律关系主体之间的人身和（或）财产及（或）行为进行的某种安排, 作为规范商事活 动的合同本身具有很强的任意性并较容易在各国形成统一认识，但有些与人的身份、能力、 婚姻、家庭、继承及某些特定行为（如奢博、卖淫嫖娼）有关的民事领域达成的协议则受一 国法律传统的影响, 各国之间实体法规定内容差异较大不宜允许当事人进行法院的选择和法 律的选择。而中国《民事诉讼法》及其司法解释、中国缔结的双边司法协助条约和《涉外民 事关系法律适用法》及其司法解释对法院选择并未更细化地限定允许当事人协议选择的范围, 对于当事人协议选择法律的范围尤其是合同领域法律选择的规定，也没有排除某些特殊的民 事内容。通过将可以协议管辖的民事领域范围更加细化, 参照海牙《协议选择法院公约》第 二条立法的模式, 通过列举的方式列出禁止当事人协议管辖的事项, 并对特定合同领域法律 选择的范围施加限定性条件，使之与中国公共秩序保持一致。

通过对协议选择法院和协议选择法律的相关内容进行限, 可使国际民商事纠纷解决各部分具 体制度保持一致。只要符合中国协议管辖的范围, 和法律选择的范围, 即便最终适用的实体 
法与中国的法律规定内容不一致, 也予以承认和执行, 而对当事人的法院选择和法律选择领 域所作的限制, 则能保证最终外国法的适用和外国判决的承认与执行将不会与中国法律的基 本原则或国家主权、安全、社会公共利益形成真正的冲突。

\section{6 结论}

调整限制协议管辖的范围比过多地适用公共秩序保留更容易使各国法律冲突平顺解决，且不 易使各国之间就此产生外交争端。当中国法院审查外国判决时，如根据中国的诉讼法及冲突 法允许当事人通过协商一致进行法院及法律适用进行选择时, 不宜再以公共秩序保留为由拒 绝该外国判决的承认与执行。公共秩序作为一国法律秩序的 “安全阀” ，应该是形式意义大 于实用价值, 相关法律规定越完善, 公共秩序保留制度的应用机会将越少。为使意思自治与 公共秩序保留制度和谐共生, 则应在当事人意思自治的范围及内容设计上预留与公共秩序的 应用空间。

\section{Acknowledgements}

The study is supported by 2017 Tianjin Administration of Justice System Key Research Project — Exploration and Thinking of our Foreign Legal Services with the Belt and Road (Grant No. 2017016).

\section{References}

[1]. Renshan Liu. The Principle of Judicial Courtesy in the Recognition and Enforcement of International Civil and Commercial Matters - A Study of the Related Theories and Practices of Britain and Canada [J]. China Legal Science. Vol. (2010) No. 5, p. 63-75.

[2]. Yongmei Ma. The Public Order in the Recognition and Implementation of Foreign Judgments [J]. Tribune of Political Science and Law. Vol. (2010) No. 5, p. 62-67.

[3]. Keyu Wang. A comparative Study of the Due Process under Brussels System and Hague Convention System-Based on the Recognition and Implementation of Foreign Judgments [J]. Journal of Comparative Law. Vol. (2009) No. 3, p. 114-122.

[4]. Yitong Liu. On the Examination Rules of Indirect Jurisdiction [J]. Citizen and Law. Vol. (2015) No. 1, p. 11-14.

[5]. Weigong Xu. The Application of the Principle of Autonomy in the Choice of Law in China [J]. Law Science. Vol. (2013) No. 9, p. 24-35. 